\title{
Die Universalität der Transzendenz. Systematische Thesen
}

Thomas Rentsch

The universality of transcendence can be shown in three fundamental dimensions: The ontological and cosmological transcendence, the existence of human beings, the existence of language. Only together do these dimensions constitute the conditions of possibility of human life and its sense. Religious traditions can be understood as cultural forms of reflection on the dimensions of transcendence. Philosophy of religion can be developed as critical hermeneutics of transcendence.

Thomas Rentsch is Professor for Practical Philosophy and Ethics at Dresden University of Technology.

1. Es geht philosophisch zunächst darum, einen fundamentalontologischen Transzendenzbegriff zu entwickeln, der weder auf objektivistische, verdinglichte und hypostasierte, noch auf religiöse und ungeklärte metaphysische Transzendenzverständnisse rekurriert, der es aber gerade so ermöglichen soll, über kritisch-hermeneutische Methodik den Prozess des - kulturell und geschichtlich außergewöhnlich komplexen - Reflexivwerdens der Transzendenzdimensionen zugänglich und explizierbar werden zu lassen. Als zentrale Züge der Transzendenzdimensionalität lassen sich zunächst Unverfügbarkeit und wesentliche Entzogenheit feststellen, die sich näherhin als lebenssinnkonstitutive Negativität verstehen lassen.

2. Was ist Transzendenz, wie lässt sie sich verstehen? Worin gründet sie? Die Klärung dieser Fragen soll unabhängig von den vielfach mit diesem Begriff unmittelbar assoziierten Bereichen sowohl der Religion und Theologie als auch der Metaphysik erfolgen. Diese Klärung muss daher noch grundsätzlicher, fundamental-anthropologisch ansetzen und die Konstitution der Transzendenz formal und strukturell explizieren. Zudem ist diese Klärung negativ-kritisch zu verstehen, da sie sich methodisch nur ontologie-, erkenntnis- und sprachkritisch durchführen lässt. Nur so ist es möglich, die konstitutive Selbstreflexivität der Transzendenzthematik methodologisch von vornherein in die Analyse einzubeziehen, anstatt ungeklärt von objektivistischen, verdinglichten und hypostasierten Transzendenzverständnissen auszugehen, wie dies vielfach geschah und geschieht. So kann es auch gelingen, das Verhältnis von Transzendenz und Immanenz ohne vorgängige metaphysische Prämissen und ohne einen vorgängigen antithetischen Dualismus zu klären. Demgegenüber gilt es, systematisch und kritisch-hermeneutisch einen 
komplexen Differentialismus der Modi, Formen und Aspekte von Transzendenz auf allen Ebenen der gesellschaftlichen und kulturellen Praxis zu erreichen, um so kulturelle Paradigmen der Transzendenz zum Beispiel in den Bereichen der Moral, der Ethik, des Rechts und der Politik, in denen der Kunst, der Architektur, der Dichtung, der Philosophie und auch der Religionen, Mythen und Theologien aufzuweisen und zu analysieren.

3. Nur so ist es methodisch möglich, eine Architektonik und Topik zu entwickeln, die hermeneutisch die jeweilige Kontextualität der Transzendenzverständnisse und Praxen möglichst genau berücksichtigt, ohne dabei reduktionistische Unterbestimmungen oder unkritische Dogmatismen zu implizieren. Es gilt dann zu fragen: Wie artikuliert sich jeweils der für das Selbstverständnis und die Identität von Individuen, Gemeinschaften und Gesellschaften sinnkonstitutive Transzendenzbezug? Wie gestalten sich die auf ihn ausgerichteten kommunikativen Lebens- und Praxisformen, die kulturellen Paradigmen? Mit dieser mehrfachen Selbstreflexivität steht auch die irreduzible Verbindung von Transzendenz und normativer, sprachlich und praktisch gegründeter Intersubjektivität im Zentrum der Analyse.

4. Um die Ansprüche der soeben skizzierten Transzendenzanalyse systematisch einzulösen, habe ich in Untersuchungen der letzten Jahre zu einer kritischen Religionsphilosophie, zur Gottesfrage und zum Verständnis von Transzendenz eine Prototheologie entwickelt. ${ }^{1}$ Der methodische Anspruch dieser Prototheologie ist es, diejenigen Phänomene („Urphänomene“) einer menschlichen Welt freizulegen und aufzuzeigen, die für religiöse, theistische und zum Beispiel auch mystische Welt- und Selbstverhältnisse grundlegend sind. Dieser Aufweis aber erfolgt selbst dem Anspruch nach neutral, formal, strukturell, anders gesagt: phänomenologisch-deskriptiv. In der Terminologie der Phänomenologie Heideggers kann hier von formaler Anzeige gesprochen werden, mit Wittgenstein von dem, was sich zeigt. Die prototheologischen Urphänomene sind die fundamentalontologischen Transzendenzdimensionen. Sie seien im Folgenden aufgewiesen.

5.1. Die erste Transzendenzdimension nenne ich die ontologisch-kosmologische Transzendenz. Sie meint die Existenz der Welt, mit Heidegger das Dass des Seins des Seienden bzw. das Wunder aller Wunder, dass Seiendes ist. Wir können diese Dimension aber auch mit Bezug auf das Universum, das All bzw. den Kosmos mit seinen Milliarden Galaxien erläutern. Unfassbar ist nicht nur die Größe dieser Dimension, sondern schlechthin unfassbar ist ihre bloße Existenz. Auch wenn in der modernen Physik Ansätze zur „Erklärung“ wie die sogenannte „Urknall“Hypothese entwickelt werden - die irreduzible Vorgängigkeit, Unerklärlichkeit

1 Vgl. Rentsch 2011. 
des Urphänomens, dass überhaupt etwas ist, und nicht nichts (Leibniz), bleibt stets erhalten und ist als Sinnhorizont von allem, was überhaupt denkbar, erfassbar, erklärbar ist, unhintergehbar. Wir können diese Unhintergehbarkeit philosophisch sowohl fundamentalontologisch als auch transzendental artikulieren. Erkenntniskritisch ist Kant Recht zu geben: Wir werden aufgrund unserer Endlichkeit nie den Ursprung bzw. seinen Grund erkennen können, welche Konstruktionen die Kosmologie auch noch entwirft, seien es „Paralleluniversen“ oder Vorgänge vor dem „Urknall“. Aber diese Grenze unseres Erkennens ist, so zeigt sich, in eins sinnkonstitutiv. Es zeigt sich daher bereits bei dieser ersten Transzendenzdimension: Ihre Vorgängigkeit ist schlechthin sinnermöglichend, sowohl oder gerade weil sie völlig unverfügbar und uns entzogen ist. Diese Struktur nenne ich sinnkonstitutive Unverfügbarkeit bzw. sinnkonstitutive Negativität. Das heißt auch: Die Transzendenzdimension ist weder ein „Gegenstand" noch irgendwie objektivierbar, und dennoch ist sie stets und immer ,da“, das heißt, sie ist im Alltag, in der Lebenswirklichkeit, in jedem Lebensvollzug, im Schlaf und Traum immer gegenwärtig, und dies, ob wir uns dessen bewusst sind oder nicht. Transzendenz ist verborgen, entzogen, und dennoch ständig sinnermöglichend. Traditionell würde man sagen: Transzendenz ist gleichsam wirklicher als die Wirklichkeit, ens realissimum.

Ferner ist diese Transzendenzdimension auch keineswegs statisch objektivierbar; vielmehr ereignet sie sich ständig prozessual, und dies seit unvordenklicher Zeit. Ihre „Wirklichkeit“ vor aller immanenten Kausalität lässt sich daher mit den traditionellen Grundbegriffen der creatio ex nihilo und der creatio continua auszeichnen. Denken wir die ontologisch-kosmologische Transzendenzdimension prozessual, so wird ein weiterer Aspekt dieses Urphänomens deutlich: Das authentische Wunder des unerklärlichen Dass des Seins des Seienden betrifft nicht nur das Universum als Ganzes, sondern jedes auch noch so kleine hervorgehende Seiende bis zu atomaren Mikrostrukturen. Es zeigt sich, dass sich das Dass in der konkreten Wirklichkeit „intern unendlich“ (Hegel) verdichtet, das heißt: Es individuiert sich. Dieser Prozess der Individuation führt zu konkreten Einzelphänomenen, den Individuen. Auch diese sind letztlich unfassbar: Individuum est ineffabile (Goethe). Das principium individuationis artikulierte diesen Aspekt der ersten Transzendenzdimension in der Tradition. Wir können sagen: Die absolute Transzendenz der konkreten Wirklichkeit in ihrer prozessualen Individuierung gilt für alles Singuläre. Wir können zwar stets viele Aspekte eines Individuums (einer Blume, eines Steins, eines Tieres) ,abschatten“ und erkennen. Aber: Das Ganze jedes Individuums bleibt stets und immer unfassbar. Es ist uns konstitutiv entzogen und dauerhaft unerkennbar. Und dennoch: Dies ist die wahre Wirklichkeit, transpragmatisch und transrational, sinnkonstitutiver Grund, vorgängig und für alles Erkennen sinnkonstitutiv. Das heißt: Die erste Transzendenzdimension zeigt sich an jedem Phänomen, an jedem Individuum und in jedem Augenblick, ob uns dies nun bewusst ist oder nicht. Diese Analyse zeigt auch mit und gegen Kant, dass dessen Fundamentalunterscheidung von mundus 
sensibilis und mundus intelligibilis sich nicht antithetisch-dualistisch verstehen lässt, wie Kant selbst es nahelegt. Vielmehr sind Immanenz und Transzendenz intern unendlich dicht verschränkt. Wir können hier auch vom „Geheimnis der Wirklichkeit" (Ebeling) sprechen.

5.2. Die zweite unvordenkliche Transzendenzdimension ist die Existenz des Menschen, vorgängig bereits des Lebens überhaupt. Ich bezeichne diese Dimension näherhin als die interexistentielle und existentielle Transzendenz. Es gilt zu sehen: Ich bin mir selbst letztlich unverfügbar, habe mich nicht geschaffen, bin mir vorgegeben, bis ich dann - nur auf dieser Basis - bestimmte autonome Praxisformen zur Gestaltung meines Lebens entwickeln kann. Dass ich überhaupt bin, das geht strikt aller mir möglichen Sinnerfahrung bereits voraus und ermöglicht diese überhaupt erst. Wiederum haben wir es mit einem rationalen, ganz konkreten Wunder zu tun, ebenso mit der Struktur der sinnkonstitutiven Entzogenheit, Unverfügbarkeit, transpragmatischen Negativität. Und ferner: Diese existentielle Transzendenz wird noch dadurch radikalisiert und fundiert, dass sie ihrerseits noch ermöglicht wird durch die transpragmatische Interexistentialität. Was heißt das? Ich bin nur wirklich geworden durch meine Eltern, Großeltern, Vorfahren. Auch die leibliche und kommunikative Interexistenz ist und bleibt entzogen und absolut sinnkonstitutiv. Das gilt auch in der gesamten Lebenspraxis. Ich bin - wie jeder und jede Andere - bleibend angewiesen auf die Mitmenschen: auf meine Eltern, die Lehrer, die Ärzte und Helfer, auf alle sozialen, rechtlichen und politischen, ökonomischen Organisationsformen, die letztlich immer von den Mitmenschen, von ihrer Arbeit und Leistung getragen werden. Ich lebe mit und durch sie. Im Bereich der Freundschaft, der Partnerschaft und Liebe, die evident wesentlich sinnkonstitutiv sind, wird zudem wiederum deutlich: Diese authentischen Formen kommunikativer Interexistentialität, der lebenstragenden Mitmenschlichkeit, sie sind ermöglicht gerade durch die sinnkonstitutive Entzogenheit, die praktische, transpragmatische Transzendenz aller Mitmenschen. Existentielle und interexistentielle Transzendenz konstituieren die humane Welt, und dies ständig in aller Alltäglichkeit: in den praktischen Lebensformen des Zuhörens, der Hilfe, des Sich-Beratens, des Denkens an den Anderen, in Freundschaft und Liebe. Wahrhaftigkeit, Aufrichtigkeit, Vertrauen, - sie werden ermöglicht durch interexistentielle Transzendenz. Es ist aber wiederum gerade die wechselseitige wie auch die individuelle Nichtobjektivierbarkeit unserer Existenz - eben deren Transzendenz -, die unsere personale Integrität und die Perspektive autonomen Handelns eröffnet und ermöglicht. (Hier ist auch der Ort der Freiheitsanalyse Kants.) Der Sinngrund unserer personalen Freiheit und Würde ist nur negativ erfassbar: als die funktionale und kausale Unableitbarkeit unseres Handelns, als die deterministische Uneinholbarkeit unseres zukünftigen Handelns. Die Rede von der „Unantastbarkeit“ des Menschen in seiner Würde artikuliert diesen Transzendenzaspekt. Es ist dieser Transzendenzaspekt, der die unbedingte Achtung und Anerkennung im Mitsein mit den Anderen als Personen 
mit irreduzibler Würde konstituiert. Ebenso ermöglicht dieser Aspekt ein authentisches Selbstverhältnis in Freiheit. Alle funktionalen, kausalen, deterministischen, reduktionistischen Thematisierungen der menschlichen Praxis setzen bei genauer Betrachtung die personale Autonomie bereits implizit methodisch voraus, und sie gründet in der existentiell-interexistentiellen Transzendenzdimension.

5.3. Die dritte Transzendenzdimension ist die der Sprache, die Transzendenz des Logos. Sie ermöglicht erst Sinn, Bedeutung, Wahrheitsansprüche und so vernünftige Kommunikation. Auch diese Transzendenzdimension, so „immanent“ sie auch erscheinen mag, geht mir und allen Menschen im strengen Sinn stets voraus. Wir kommen zu uns und werden zu uns selbst in, mit und durch die Sprache, haben sie weder gemacht, noch können wir sie gänzlich erfassen und objektivieren. Wir können noch nicht einmal den Gebrauch eines Wortes (zum Beispiel „rot“) insgesamt überblicken. Auch im Blick auf diese Transzendenzdimension sind keine mystischen Sondererfahrungen oder quasi-mythischen Entwürfe nötig, um die Transzendenz des Logos zu explizieren. Dass - und wie - wir sprechen können, ist eine unerklärliche, uns vorgängige Bedingung der Möglichkeit und Wirklichkeit unserer humanen Welt. Zu allem Erklären, ja zu allem Erkennen benötigen wir die Sprache bereits. Gleichwohl ist das Wunder der Sprache alltäglich, jedermann bekannt und universal zugänglich wie auch die Transzendenz des Seins und aller Wirklichkeit, wie auch unsere eigene Existenz. Die Sprache ermöglicht zuallererst unsere freie Selbstentfaltung und individuelle Selbstbestimmung, ohne sie gibt es kein Weltverständnis und keine Selbsterkenntnis. Die Wirklichkeit der Sprache erschließt uns fundamentale, lebenssinnkonstitutive Horizonte wie den der Einheit, des Wahren, des Guten und des Schönen. (Hier ist der Ort von Platons Ideenlehre und der Scholastischen Transzendentalienlehre.) Ohne dass wir ganze Sätze in ganzen Lebenssituationen formulieren und begreifen können, ist unsere humane Existenz undenkbar. Weder ein Sinn von Sein noch eine humane Welt wären ohne unsere kommunikative Selbsttranszendenz auch nur möglich. Die Sprache jedoch ermöglicht ständig innovative Sinneröffnung. Mit dem - theoretisch unerklärlichen - Hervorgang der sprachlichen Transzendenzdimension in die Wirklichkeit unserer Welt ist irreduzibler Sinn konkret geworden und wird ständig weiter konkret. Dieses prozessuale Transzendenzgeschehen ermöglicht und trägt unser eigenes Selbstbewusstwerden und Transzendieren. Dieses Geschehen ist - wie die anderen Transzendenzdimensionen - in der lebensweltlichen Alltäglichkeit verborgen, anwesend-abwesend. Auch die Sprache gehört zu den nicht-objektivierbaren Sinngründen unseres gesamten Seins, zu den transpragmatischen Sinnbedingungen unserer Existenz.

6. Die soeben skizzierten Transzendenzdimensionen sind ersichtlich universal, sie „gehören“ keiner Theorie, keiner Religion, keiner Kultur, keiner Philosophie, keiner geschichtlichen Epoche. Wichtig ist zunächst zusätzlich, dass sie - mit 
Husserl und Heidegger gesprochen - gleichursprünglich sind (,,äquiprimordial“): Sie sind irreduzibel aufeinander, unableitbar voneinander und nur durch und miteinander analysierbar und verstehbar. Nur im Horizont und Medium aller dieser Transzendenzdimensionen lässt sich auch nur eine konkrete Lebenssituation verstehen, wie sie sich alltäglich ereignet. Aus diesem Konstitutionsaspekt ergibt sich die Perspektive eines Holismus der Transzendenz, die ich hier nur andeute. Ebenso lässt sich aus den bisherigen Analysen eine Mitte bzw. ein Zentrum der Transzendenzdimension auszeichnen: Dieses Zentrum ist die existentielle Individualität des Menschen, weil nur durch sie die anderen Dimensionen bewusst werden können.

7. Die bisherige Analyse zur Universalität der Transzendenz kann so geprüft und differenziert begründet werden, dass auf ihrer Grundlage eine Topik der Modi kultureller Paradigmen der Transzendenz erarbeitet wird. In dieser Topik muss gezeigt werden, dass und wie die aufgezeigten Transzendenzdimensionen in den Mythen, in den Weltreligionen, in den mystischen Traditionen, in der Philosophie, aber auch in der Kunst (und zum Beispiel auch in der säkularen Dichtung und Literatur) thematisiert, vergegenwärtigt und gestaltet werden. Ich bezeichne diesen Prozess als das kulturelle Reflexivwerden der Transzendenzdimensionen. Es wird konkret deutlich in den Schöpfungstheologien, aber auch in den komplexen Meditationspraxen der Religionen, in den neuplatonischen Henologien und Kosmologien, in den Traditionen der Heiligung, aber auch in der Porträtkunst und in der Landschaftsmalerei, um nur einige Beispiele zu nennen. Auf diese Weise kann eine kritische Hermeneutik der Transzendenz (und ihrer Universalität) in ihrer ganzen Komplexität entwickelt werden, und zwar inter- und transkulturell.

\section{Literaturverzeichnis}

Rentsch, Thomas: Gott. Berlin - New York 2005.

Ders.: Transzendenz und Negativität. Religionsphilosophische und ästhetische Studien. Berlin - New York 2011.

Ders.: „Transzendenz - Konstitution für die okzidentale Rationalität“, in: Marion, JeanLuc / Schweidler, Walter (Hg.): Christentum und Philosophie. Einheit im Übergang. Freiberg - München 2014, S. 137-147. 